\title{
Distance, symmetry, and task affect right-left vs. up-down judgments
}

\author{
RUTH H. MAKI and BARBARA A. HOLZER \\ North Dakota State University, Fargo, North Dakota 58105
}

\begin{abstract}
Subjects judged whether a stimulus in a designated location pointed right, left, up, or down, or they judged whether two right-left or up-down stimuli pointed in the same or different directions. Pairs of stimuli were either far apart or close together, both of the same form or not, and aligned horizontally or vertically. In the location task, the only significant effect was that far stimuli were responded to faster than near stimuli, probably because selective attention was easier. In the same-different task, stimulus orientation interacted with alignment such that vertical stimuli were faster with horizontal alignment and horizontal stimuli were faster with vertical alignment. This effect occurred, however, only when stimuli were of the same form and when they had opposite orientations. It was attenuated when they were far apart. Calling symmetric stimuli different was the source of difficulty in the same-different task. There was no evidence for an inherent difficulty with right and left in either task.
\end{abstract}

A number of experiments have shown that making judgments about the location and orientation of rightleft stimuli takes longer than making such judgments about up-down stimuli (e.g., Braine, Relyea, \& Davidman, 1981; Fisher, 1979; Maki, Grandy, \& Hauge, 1979; Maki, Maki, \& Marsh, 1977; Rudel \& Teuber, 1963). However, under some conditions, this right-left difficulty is not observed. One such condition involves a successive discrimination task in which subjects make a response to only one stimulus on a dimension. Although Farrell (1979) reported that responding to right or left took longer than responding to up or down. Maki (1979) replicated his experiment and found no difference. Further support for the conclusion that there is no right-left difficulty when a single stimulus is presented came from a similar experiment by Sholl and Egeth (1981). They also reported no difference between right-left and up-down in such a successive discrimination task. In an experiment with children, Fisher (1979) found that there was no difficulty in discriminating right from left when each stimulus was presented alone but right and left were very difficult when they were presented simultaneously.

While there seems to be no right-left difficulty when single stimuli are presented to adults or children, simultaneous discrimination tasks in which two stimuli are presented together produce varying results. As shown by both Fisher (1979) and by Rudel and Tueber (1963), children have much difficulty with right and left in such tasks, but this difficulty may be modulated when stimuli are presented vertically rather than horizontally (Huttenlocher, 1967). With adults, most experiments in

The authors wish to thank Joel Bakken for helping to test the subjects. Reprint requests should be sent to the first author at the Department of Psychology, North Dakota State University, Fargo, North Dakota 58105. which two stimuli are presented together have employed a same-different task and reaction time (RT) has been measured. Sometimes right-left stimuli take longer than up-down stimuli, and sometimes they do not. One pattern of results observed with the same-different task is exemplified by results found by Wolff (1971). When the stimulus pairs were aligned horizontally, right-left stimuli took longer than up-down stimuli; when they were aligned vertically, up-down stimuli took longer than right-left. A similar pattern was observed by Sekuler and Houlihan (1968), but the slower responses to updown than to right-left stimuli with the vertical alignment was not significant, whereas faster responding to up-down than to right-left with the horizontal alignment was significant. Sekuler and Houlihan concluded that the right-left task is generally more difficult than the up-down task, although the size of this effect can be modulated by alignment. A study by Corballis, Miller, and Morgan (1971) reported different results. All of their stimuli were presented horizontally, so right-left stimuli should have taken longer than up-down stimuli. However, there was no difference between the two types of stimuli. There is also one example in the literature in which a successive version of this same-different task produced longer RTs to right-left than to up-down stimuli. Butler (1964) presented T-shaped stimuli that were oriented up, down, right, or left. One stimulus was presented and a second stimulus was presented in the same location $3 \mathrm{sec}$ later; subjects were to decide whether the two stimuli were same or different. Rightleft orientations took longer than up-down orientations.

The present experiment was designed to clarify the conditions under which right-left and up-down stimuli produce different RTs. Because much of the literature with children has involved a task in which the child pointed to one of two stimuli, a similar task in which 
RT could be measured was devised for adults. Half of the subjects participated in a location task in which a block of light indicated one of four locations on a terminal screen. Two stimuli then appeared, one in the designated location and one above, below, to the right of, or to the left of it. During a block of trials, subjects were to respond only if one specific stimulus was in the designated location and not if the other stimulus was there. The other half of the subjects participated in a same-different task in which they responded only if the two stimuli were the same or different during a block of trials.

The distance between pairs of stimuli was also varied. It appears that Wolff's (1971) stimuli were close together (1 deg $42 \mathrm{~min}$ ), whereas Corballis et al.'s (1971) stimuli were fairly far apart $(4 \mathrm{deg})$. When stimuli were aligned horizontally, Wolff found that right-left took longer than up-down, but Corballis et al. did not. Butler (1964), who presented pairs successively, also found that rightleft stimuli took longer than up-down stimuli. Perhaps, presenting stimuli in exactly the same location, even though they are separated by time, is similar to presenting them close together in space. The present experiment, then, was designed to determine whether or not distance might explain some of the earlier discrepancies in the literature.

The mirror-image nature of the stimuli was also manipulated between subjects. Corballis and Beale (1976) have argued that right and left stimuli are difficult to discriminate because of the bilateral symmetry of the nervous system, although they do not believe that the simultaneous tasks used in the present experiment involve right-left discrimination. However, others (e.g., Sekuler \& Houlihan, 1968) have argued that the simultaneous task relies on right-left discrimination and that the mirror-image nature of the stimuli is important. If right-left judgments are more difficult than up-down judgments because of the bilateral symmetry of the nervous system, then this problem should be attenuated if pairs of stimuli are not mirror images. Half of the subjects in the present experiment saw two stimuli of the same type on each trial: two arrowheads or two horseshoes. The other half of the subjects saw one stimulus of each type on each trial: one arrowhead and one horseshoe. Thus, subjects in the constant-stimulus condition saw mirror-image stimuli, at least on some trials, but subjects in the mixed condition never saw mirror images. If the mirror-image nature of the stimuli is important, then the pattern of results should differ for the two types of stimuli.

The experiment, then, consisted of four groups of subjects defined by the factorial combination of task and constant vs. mixed stimuli. For all subjects, pairs of stimuli were near or far, right-left or up;down, and aligned horizontally or vertically. In the same-different task, it was expected that up-down vs. right-left stimuli would interact with alignment, at least when they were close together and when they were constant on all trials. Whether similar effects would occur in the location task and when stimuli were far apart or mixed could not be predicted from past results.

\section{METHOD}

\section{Subjects}

Arrowheads and horseshoes were constructed from seven asterisks and displayed on an ADM 3A terminal connected to an Automated Data Systems 1800E minicomputer. The outermost three asterisks were located in the same position for the two types of stimuli; the pointed or curved appearance was formed by moving the four inner asterisks. The up and down stimuli were $3.51 \mathrm{~cm}(9 \mathrm{deg} 36 \mathrm{~min})$ wide and $2.2 \mathrm{~cm}(6 \mathrm{deg}$ $0 \mathrm{~min}) \mathrm{high}$; the right and left stimuli were $1.89 \mathrm{~cm}(5 \mathrm{deg}$ $12 \mathrm{~min})$ wide and $3.85 \mathrm{~cm}$ (10 deg $38 \mathrm{~min})$ high. Vertical and horizontal stimuli of exactly the same dimensions could not be constructed on the terminal. Because of the characteristics of spacing on the terminal, the exact distance between pairs of stimuli also varied somewhat, depending upon orientation and alignment. Near pairs of stimuli averaged $1.1 \mathrm{~cm}(3 \mathrm{deg} 7 \mathrm{~min})$ apart, and far displays averaged $4.1 \mathrm{~cm}$ (11 deg $27 \mathrm{~min}$ ) apart.

\section{Procedure}

One group of subjects performed the location task, in which a block of light (the cursor) appeared on the terminal for $2 \mathrm{sec}$ to indicate the location for that trial. Two stimuli appeared, and subjects were supposed to say "yes" into a microphone attached to a voice-activated relay if the stimulus in the location designated by the cursor was the one to which they were to respond on that block of trials. The designated location was always at the top, bottom, left, or right of the screen, and one stimulus always appeared in that location. The other stimulus was always from the same dimension; that is, pairs were always up and down or right and left. The order of correct stimuli was balanced across subjects such that up, down, right, and left stimuli were responded to equally often across subjects in each of the four blocks of trials.

Another group of subjects performed the same-different task. For them, the cursor always appeared in the center of the screen for $2 \mathrm{sec}$ as a warning signal. Subjects said "yes" into the microphone attached to the voice-activated relay if the two stimuli were oriented either the same way or in different ways. Across four blocks of trials, each subject said "yes" to up-down same, up-down different, right-left same, and right-left different pairs. The order in which subjects responded to these stimuli was balanced across subjects so that all types of pairs were responded to in each block equally often.

In each task condition, half of the subjects saw only one type of stimulus (arrowhead or horseshoe) on all trials and the other half saw one stimulus of each type on all trials. In the location task, subjects responded to the orientation of the stimulus in the designated location whether the stimuli were constant or mixed. Likewise, in the same-different task, subjects indicated whether the orientation of the stimuli was same or different for both constant and mixed stimuli.

Before the beginning of each of the four blocks of 64 trials, instructions indicated which stimulus should be responded to in that block of trials. No verbal description of orientation or samedifferent was given; subjects saw examples of the correct stimuli. Ten practice trials were presented. If a subject made more than two errors on the 10 practice trials, the instructions were represented and 10 more practice trials were given. If correct responses were given on 8 or more of the trials, the 64 data collection trials were presented. Subjects should have responded "yes" on half of the trials (the "correct" stimulus was in the designated location or the two stimuli were oriented the same or 
differently, whatever the subject was supposed to respond to). On the other half of the trials, no response should have been made because the "wrong" stimulus was in the designated location or because the stimuli were different if the subject was responding to "same" or the same if the subject was responding to "different." RT was recorded by the computer on all positive trials. The stimuli were erased following a response. On negative (no-response trials), there was simply a 2-sec interval during which the computer checked for a response. All trials were then followed by a 2-sec intertrial interval. Sessions took approximately $45 \mathrm{~min}$.

\section{Subjects}

Subjects were volunteers from introductory psychology classes at North Dakota State University who participated for extra credit toward their course grades. Twelve subjects were randomly assigned to each of the four groups defined by the factorial combination of task and constant vs. mixed stimuli. One subject in the same-different mixed condition was replaced because she did not respond on over half of the correct trials during two blocks.

\section{RESULTS AND DISCUSSION}

\section{Location Task}

Mean RTs for the location task were analyzed in a mixed analysis of variance with constant or varied stimuli as a between-subjects variable and orientation, alignment, and distance as within-subjects variables. The only significant effect was longer RTs for near stimuli (mean $=424 \mathrm{msec}$ ) than for far stimuli (mean $=401 \mathrm{msec}$ ) $[\mathrm{F}(1,32)=34.77, \mathrm{MSe}=778.34, \mathrm{p}<.05$, the significance level to be used throughout this article]. Because subjects needed to respond to only one of the two stimuli in this task, they had to direct their attention to the stimulus in the designated location. Such selective attention must have been easier when the two stimuli were farther apart. The ease of attending to one of two stimuli was tapped by this location task, but the task showed no effects of orientation or alignment. Up-down stimuli produced RTs of $415 \mathrm{msec}$ when they were aligned hroizontally and $409 \mathrm{msec}$ when they were aligned vertically. Right-left stimuli produced RTs of $416 \mathrm{msec}$ for horizontal and $409 \mathrm{msec}$ for vertical alignment. Clearly, the Orientation by Alignment interaction was not significant $(F<1)$, nor did this effect interact with other variables. Deciding if one of two stimuli was oriented up or down was no easier than deciding if it was right or left, regardless of alignment. Error rates were very low in this task. Subjects did not respond on $1.25 \%$ of the correct trials, and they did respond on $1.6 \%$ of the incorrect trials.

\section{Same-Different Task}

A five-way analysis of variance including the four variables from the above analysis plus same-different orientation was conducted on the mean RTs from the same-different task. In contrast to the location task, far stimuli (mean $=621 \mathrm{msec}$ ) produced longer RTs than near stimuli (mean $=604 \mathrm{msec}$ ) $[\mathrm{F}(1,22)=17.58$, MSe $=1,698.08]$. Mixed stimuli produced longer RTs than constant stimuli $[\mathrm{F}(1,22)=8.93, \mathrm{MSe}=$
$118,841.55]$, and "same" stimuli were faster than "different" $[\mathrm{F}(1,22)=32.64, \mathrm{MSe}=21,909.60]$. These effects can be seen in Figures 1 and 2, along with the significant four-way interaction among distance, stimulus constancy, orientation, and alignment $[\mathrm{F}(1,22)=$ 9.48, $\mathrm{MSe}=2,014.82]$. In addition, the interaction among same-different, orientation, and alignment was significant $[F(1,22)=5.70, \mathrm{MSe}=4,308.94]$. In order to understand these effects, each stimulus constancy and distance condition was analyzed separately. These four conditions are shown in the four panels of Figures 1 and 2.

Figure 1 shows the mean RTs in the mixed-stimulus conditions. In both the near and far mixed conditions, the only significant effect was that "same" responses were faster than "different" responses $[\mathrm{F}(1,11)=15.96$, MSe $=18,458.26$, and $F(1,11)=19.54, \quad$ MSe $=$ $22,857.59]$. Stimulus orientation and alignment produced no significant effects. The mean RTs in the constant-stimulus conditions are shown in Figure 2. In both of the near and far analyses, the three-way interaction among orientation, alignment, and same-different

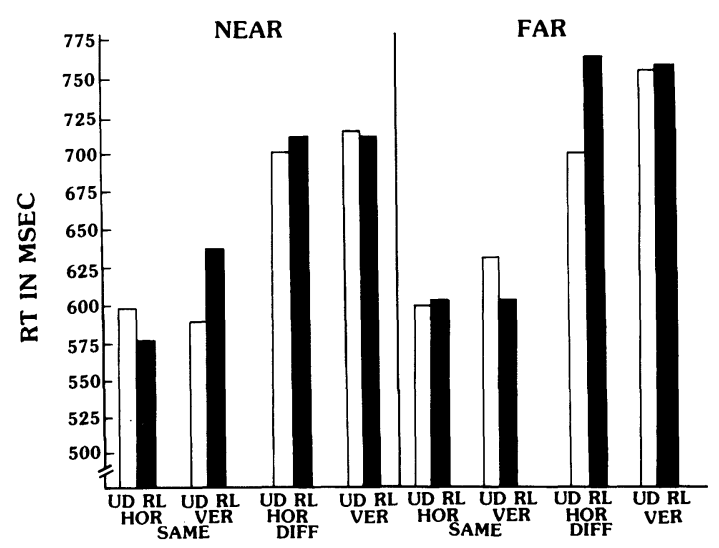

Figure 1. Mean RTs for mixed stimuli as a function of updown or right-left orientation, horizontal or veritcal alignment, same or different orientation, and near or far stimulus pairs.

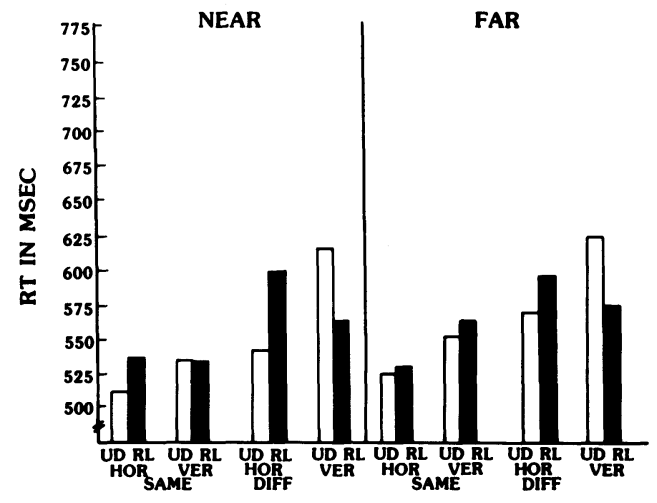

Figure 2. Mean RTs for constant stimuli as a function of up-down or right-left orientation, horizontal or vertical alignment, same or different orientation, and near or far stimulus pairs. 
was significant $[\mathrm{F}(1,11)=7.17, \quad \mathrm{MSe}=1,464.77$, and $\mathrm{F}(1,11)=15.61, \mathrm{MSe}=553.44]$. When subjects responded to "same" stimulus pairs in both the near and far conditions, orientation did not interact with alignment $(\mathrm{Fs}<1)$. However, when subjects responded to "different" stimulus pairs, orientation interacted with alignment in the near condition $[\mathrm{F}(1,11)=16.44$, $\mathrm{MSe}=2,305.98]$. With horizontal alignment, rightleft stimuli took longer than up-down stimuli, but with vertical alignment, up-down stimuli took longer than right-left stimuli. A similar trend was present in the far condition, but the interaction was slightly short of significance $[F(1,11)=4.25, \mathrm{MSe}=3,290.39]$. Error rates were low in this task. Subjects failed to respond on $1.79 \%$ of the correct trials, and they responded on $2.33 \%$ of the incorrect trials.

This experiment points to an important source of difficulty in the same-different task. There were only two conditions in which stimuli were symmetric around the axis perpendicular to their orientation: right-left different stimuli in a horizontal alignment $(<>)$ and up-down stimuli in a vertical alignment $(\stackrel{\vee}{)})$. In all other conditions, either symmetry was absent (e.g., $\wedge \vee$ ) or symmetry occurred around the same axis as the orientation (e.g.,$<$ ). Evidently, it is more difficult to call two stimuli different if they possess obvious symmetry (horizontally aligned right-left stimuli and vertically aligned up-down stimuli) than if they do not (vertically aligned right-left stimuli and horizontally aligned updown stimuli). This would produce the observed interaction between alignment and orientation, but only when two stimuli were of the same type (both arrowheads or horseshoes) and when they were oriented in different ways. In addition, there was some evidence that this effect was stronger when stimuli were closer; proximity in space probably made the symmetry more evident. The weaker effect with far stimuli might explain why Corballis et al. (1971) found no difference between right-left and up-down stimuli aligned horizontally. Their stimuli were somewhat farther apart than the near stimuli in the present experiment; this may have eliminated the difficulty with horizontally aligned right and left stimuli.

Under some conditions, it may be difficult to call two stimuli the same if they do not possess symmetry around the axis perpendicular to their orientation. Right-left stimuli that are horizontally aligned $(\ll)$ and up-down stimuli that are vertically aligned $(\hat{\lambda})$ do not possess such symmetry, yet they are to be called the same. In the present experiment, there was no evidence for such an effect with same stimuli, but in Sekuler and Houlihan's (1968) and Wolff's (1971) data, such an effect was present.

The key to understanding this same-different task seems to be the presence or absence of symmetry around the axis that is perpendicular to the stimulus orientation, whether that axis is horizontal or vertical. This finding contrasts somewhat with those of studies on the detection of symmetry (e.g., Palmer \& Hemenway,
1978), which have shown that symmetry is more easily detected around the vertical than around the horizontal axis. However, asking subjects to make samedifferent judgments as was done in the present experiment may involve processes different from those involved in asking subjects to detect symmetry.

There seems to be nothing inherently difficult about judgments of right-left as compared to up-down in either the location or the same-different task. Although symmetry is important in the same-different task, it has no relation to the bilateral symmetry of the nervous system because the symmetry effect occurred in both the horizontal and the vertical dimensions. What makes right and left more difficult than up and down in other judgment tasks (e.g., Braine et al., 1981; Maki et al., 1977; Rudel \& Teuber, 1963) evidently does not occur in these two simple discrimination tasks.

\section{REFERENCES}

Braine, L. G., Relyea, L., \& Davidman, L. On how adults identify the orientation of a shape. Perception \& Psychophysics, 1981, 29, 138-144.

ButLer, J. Visual discrimination of shape by humans. Quarterly Journal of Experimental Psychology, 1964, 16, 272-276.

Corballis, M. C., \& BeAle, I. L. The psychology of left and right. Hillsdale, N.J: Erlbaum, 1976.

Corballis, M. C., Miller, A., \& Morgan, M. J. The role of left-right orientation in interhemispheric matching of visual information. Perception \& Psychophysics, 1971, 10, 385-388.

Farrell, W. S., JR. Coding left and right. Journal of Experimental Psychology: Human Perception and Performance, 1979, $5,42-51$.

Fisher, C. B. Children's memory for orientation in the absence of external cues. Child Development, 1979, 50, 1088-1092.

HuTtenlocher, J. Discrimination of figure orientation: Effects of relative position. Journal of Comparative and Physiological Psychology, 1967, 63, 359-361.

Maki, R. H. Right-left and up-down are equally discriminable in the absence of directional words. Bulletin of the Psychonomic Society, 1979, 14, 181-184.

Maki, R. H., Grandy, C. A., \& Hauge, G. Why is telling right from left more difficult than above from below? Journal of Experimental Psychology: Human Perception and Performance, 1979, 5, 52-67.

Maki, R. H., Maki, W. S. \& Marsh, L. G. Processing locational and orientational information. Memory \& Cognition, 1977, 5, 606-612.

Palmer, S. E., \& Hemenway, K. Orientation and symmetry: Effects of multiple, rotational, and near symmetry. Journal of Experimental Psychology: Human Perception and Performance, 1978, 4, 691-702.

Rudel, R. G., \& TEuber, H.-L. Discrimination of direction of line in children. Journal of Comparative and Physiological Psychology, 1963, 56, 892-898.

Sekuler, R. W., \& Houlihan, K. Discrimination of mirrorimages: Choice time analysis of human adult performance. Quarterly Journal of Experimental Psychology, 1968, 20, 204207.

Shol, M. J., \& Egeth, H. E. Right-left confusion in the adult: A verbal labeling effect. Memory \& Cognition, 1981, 9, 339350.

WoLfF, P. Mirror-image confusability in adults. Journal of Experimental Psychology, 1971, 91, 268-272.

(Received for publication August 9, 1982.) 